\title{
Catheter ablation in an 80-year-old male with late-onset Wolff-Parkinson-White syndrome
}

\author{
Maria Silva Negroni, Sebastiano Belletti, Marco Centola, Sara Cazzaniga, Stefano Carugo \\ Division of Cardiology, San Paolo Hospital, Department of Health Sciences, University of Milan, Italy
}

Received: March 28, 2016

DOI: $10.5430 /$ crim.v3n2p50
Accepted: May 3, 2016

URL: http://dx.doi.org/10.5430/crim.v3n2p50

\begin{abstract}
Wolff-Parkinson-White syndrome is a congenital condition involving abnormal conductive cardiac tissue between atria and ventricles, that provides a pathway for a re-entrant tachycardia circuit or rapid repetitive ventricular response during atrial fibrillation. Clinical onset is usually from childhood to middle age, and symptoms vary in severity from palpitations (with or without syncope) to cardiac arrest. Presentation varies depending on the patient's age and presence of comorbidities, as arrhythmias are less tolerated in case of an associated heart disease. In individuals who do not experience symptoms, the condition may go unnoticed for years. We describe the case of an 80-year-old Caucasian male with late-onset of Wolff-Parkinson-White syndrome. He presented with a regular, hemodynamically unstable wide-complex tachycardia with left bundle branch morphology, successfully treated with medical therapy. After recurrence of tachycardia, he underwent an electrophysiological study that showed an orthodromic atrioventricular re-entrant tachycardia using a posterolateral accessory pathway and with functional left bundle branch block. Transseptal radiofrequency catheter ablation of the accessory pathway was successfully performed. After 15 months of follow-up, there was no tachycardia recurrence or findings of pre-excitation on the electrocardiogram. The treatment of both supraventricular and ventricular arrhythmias may be challenging especially in elderly patients. Catheter ablation of left accessory pathways with transseptal approach is feasible and effective in old patients with late-onset Wolff-Parkinson-White syndrome.
\end{abstract}

Key Words: Wolff-Parkinson-White syndrome, Catheter ablation, Elderly

\section{INTRODUCTION}

Accessory pathways (APs) are anomalous bands of conducting tissue that by-pass the normal atrioventricular conduction system; most commonly, they connect the atrium to the ventricle and can conduct anterogradely (from atrium to ventricle) as well as retrogradely in the opposite direction. When anterograde conduction is manifested on electrocardiogram (ECG) as a slurring of the initial portion of the QRS complex (delta wave), ventricular pre-excitation occurs because the ventricular myocardium is activated early over the AP.
The diagnosis of Wolff-Parkinson-White (WPW) syndrome is reserved for patients who have both electrocardiographic evidence of pre-excitation and symptoms suggestive of a tachyarrhythmia. Among patients with an AP, the most common arrhythmia is orthodromic atrioventricular re-entrant tachycardia (AVRT), a specific supraventricular tachycardia involving both the atrioventricular node/His-Purkinje conduction system for conduction in the anterograde direction and the AP for conduction in the retrograde direction. Supraventricular tachycardias (such as AVRT) usually have a narrow

*Correspondence: Maria Silva Negroni; Email: mariasilvia.negroni@ao-sanpaolo.it; Address: Division of Cardiology, Ospedale San Paolo, Dipartimento Scienze della Salute, Università degli Studi di Milano, Via A. di Rudinì 8, Milan 20142, Italy. 
QRS complex, but their diagnosis may be difficult in case of concomitant aberrant conduction (for example, rate-related or structural bundle branch block). WPW syndrome affects people of all ages, but it is most commonly recognized in children and young adults presenting to the emergency department (ED) with a tachyarrhythmia. ${ }^{[1]}$ Patients with WPW syndrome have an increased risk of sudden cardiac death (SCD) because an episode of atrial fibrillation (AF) with a very rapid ventricular response through the AP can lead to ventricular fibrillation. For this reason, catheter ablation (CA) of the AP became first-line therapy for WPW patients since the early 1990s; CA is also commonly performed to treat AVRT, given its high efficacy and safety. ${ }^{[1]}$ Age should not preclude invasive treatment. We report the case of an old patient with late-onset WPW syndrome successfully treated by transseptal radiofrequency $\mathrm{CA}$.

\section{Case presentation}

An 80-year-old Caucasian man called the emergency medical service for palpitations and dyspnoea during febrile illness. His medical history was significant for essential hypertension treated with atenolol $50 \mathrm{mg}$ daily. A routine ECG performed

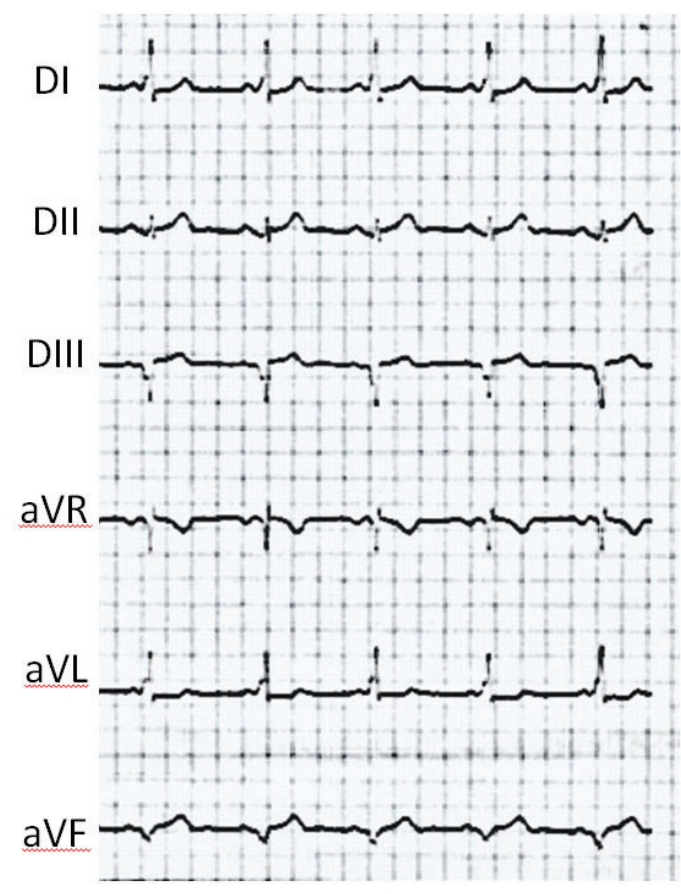

3 years earlier, at the age of 77, showed normal sinus rhythm with ventricular pre-excitation suggestive of a left-sided AP (see Figure 1). A 24-h ECG Holter monitor confirmed a permanent WPW pattern without relevant arrhythmias. At the time, he was asymptomatic for any arrhythmic event (palpitations and/or syncope). In the ED, the patient was sweaty and tachycardic, with a blood pressure of $90 / 60 \mathrm{mmHg}$. His baseline ECG showed a regular wide-complex tachycardia with a ventricular rate of 205 beats per minute (bpm). QRS width was $150 \mathrm{~ms}$, with a left bundle branch block (LBBB) morphology and a superior axis in the frontal plane (see Figure 2). The patient was successfully cardioverted with an IV amiodarone bolus (300 mg over 15 minutes). The subsequent ECG revealed sinus rhythm with normal PR interval and QRS duration, and a slight prolonged QTc interval (470 ms) (see Figure 3 ). The patient was admitted to the cardiology department and no findings of ventricular pre-excitation were observed in all subsequent ECGs. Transthoracic echocardiography showed left ventricular hypertrophy with normal ejection fraction, and a coronary angiogram (performed for a slight troponin I elevation) was normal.

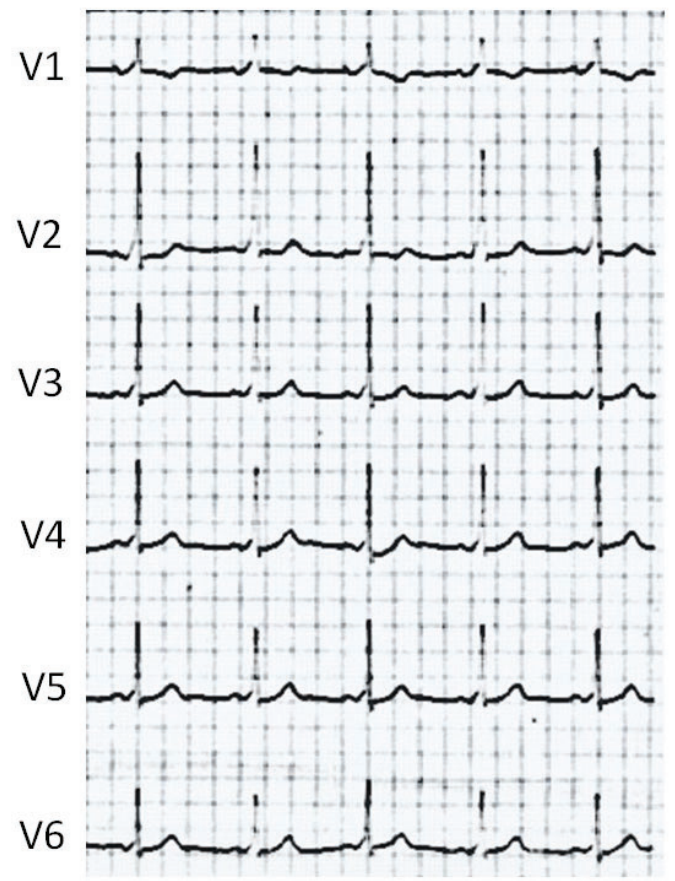

Figure 1. Twelve-lead ECG showing a pre-excitation pattern consistent with a left-sided atrioventricular accessory pathway

The patient refused further investigations and was discharged home with atenolol $100 \mathrm{mg}$ daily. Two weeks later the patient came back to the ED with recurrence of the wide-complex tachycardia. The ventricular rate was slower $(160 \mathrm{bpm})$ and it was successfully cardioverted with adenosine (6 mg IV bolus). Again, ECG after cardioversion was normal, without signs of pre-excitation. Given the history, we performed an electrophysiological study (EPS). Baseline intervals were normal, with an atrial-His (AH) interval of $70 \mathrm{~ms}$, and Hisventricular (HV) interval of $45 \mathrm{~ms}$; neither dual nodal path- 
way nor anterograde conduction through the AP were present. before. Interestingly, during the tachycardia we documented Right ventricular pacing showed an eccentric retrograde atrial activation, with the earliest atrial signal in the mid coronary sinus (CS); the AP blocked retrogradely at $390 \mathrm{~ms}$. With programmed stimulation (single extra-stimulus), a LBBB wide-complex tachycardia with a cycle length (CL) of 380 $\mathrm{ms}(158 \mathrm{bpm})$ was easily inducible and comparable to the clinical tachycardia. V-A relationship was 1:1 with an eccentric atrial activation (earliest activation in the mid CS) and a septal VA interval of $130 \mathrm{~ms}$, suggestive of orthodromic AVRT mediated by an AP localized in the left posterolateral mitral annulus (5 o'clock). The AP location was consistent with the WPW pattern on the routine ECG recorded 3 years

LBBB disappearance with concomitant acceleration of the tachycardia and VA interval reduction (167 bpm and 100 ms, respectively), the so-called Coumel sign, indicative of an orthodromic AVRT using an AP ipsilateral to the BBB. ${ }^{[2]}$ Transseptal radiofrequency CA of the AP was successfully performed (see Figure 4), with an open-irrigated catheter: at the end of the procedure, the tachycardia was non-inducible with retrograde conduction consistently occurring over the atrioventricular node. After 15 months of follow-up, the patient remained asymptomatic, without any tachycardia recurrence or signs of ventricular pre-excitation on the 12-lead ECG.

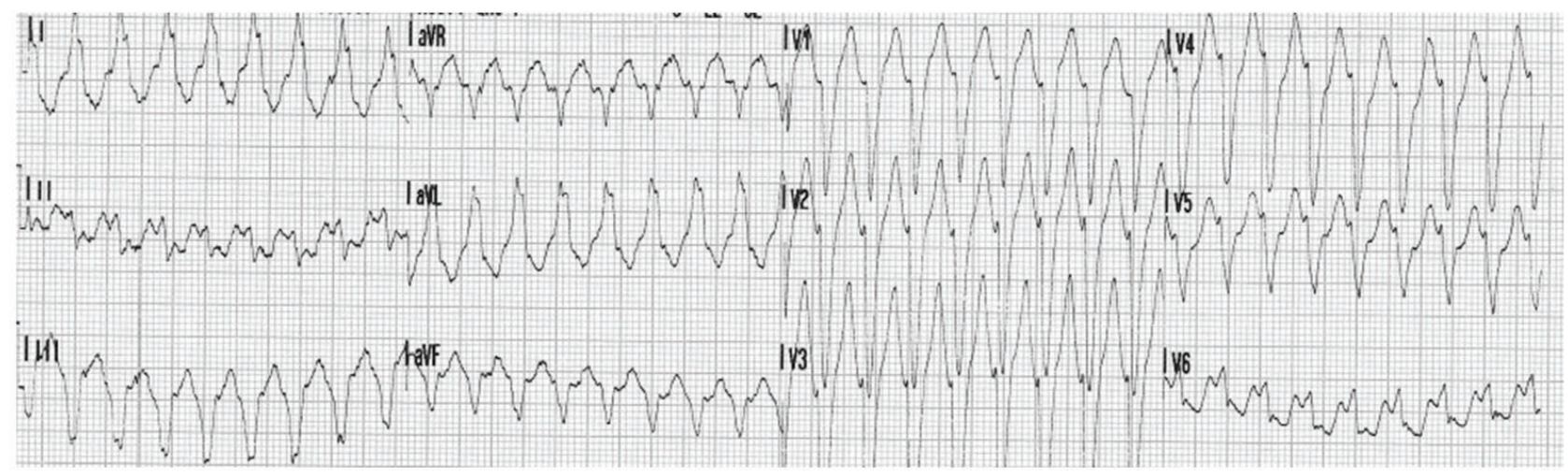

Figure 2. Twelve-lead ECG of the clinical tachycardia showing a regular wide-complex QRS tachycardia with a rate of 205 bpm
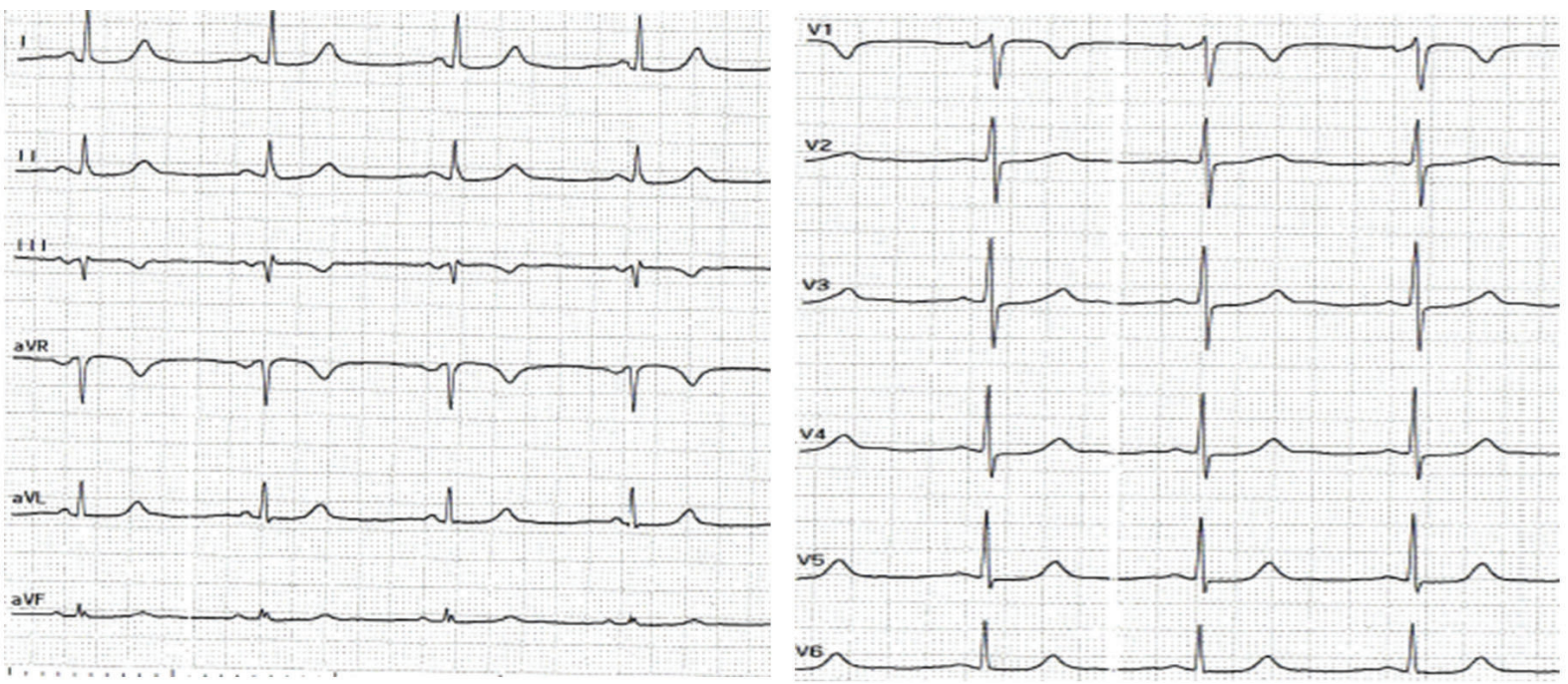

Figure 3. Twelve-lead ECG recorded after successful cardioversion to sinus rhythm showing normal atrioventricular and intraventricular conduction times 


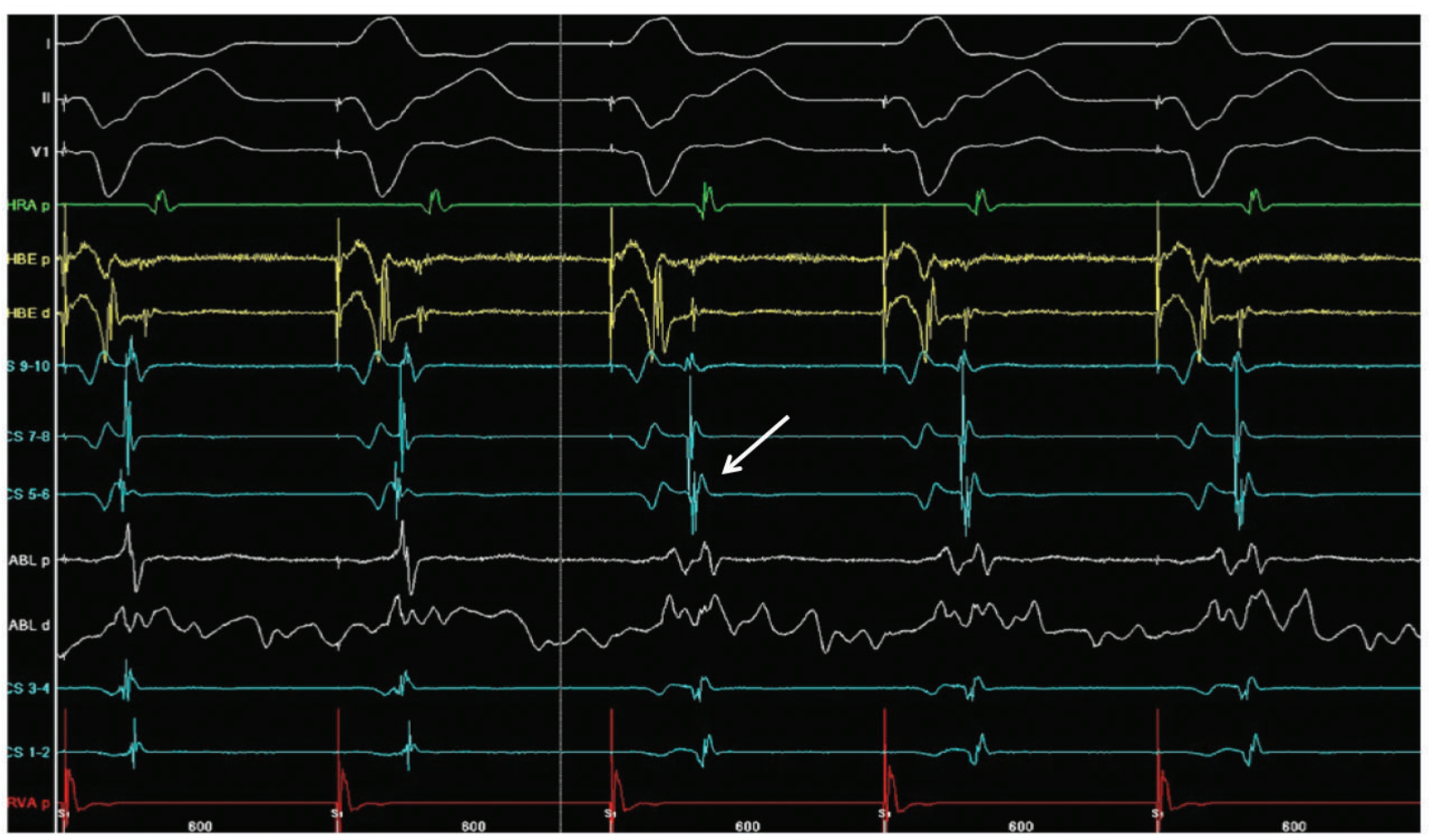

Figure 4. Simultaneous recordings at $100 \mathrm{~mm} / \mathrm{s}$ of surface leads I, II, and V1, and intracardiac electrograms of the high right atrium (HRA p), proximal and distal His bundle (HBE p, HBE d), proximal to distal coronary sinus (CS 9-10, to CS1-2) and right ventricular apex (RVA p). The earliest atrial activation is recorded on the ablation catheter (ABL p and $\mathrm{ABL}$ d) positioned at 5 o'clock over the mitral annulus; retrograde conduction over the AP is interrupted by RF ablation performed during right ventricular apex pacing (white arrow)

\section{Discussion}

The present case illustrates the occurrence and management of late-onset WPW syndrome in an elderly patient. The WPW pattern on the ECG was incidentally detected at advanced age during an outpatient visit. The WPW pattern was overt for at least 3 years but the patient was asymptomatic for over eight decades. The patient presented to the $\mathrm{ED}$ with a fast, regular, poorly tolerated wide-complex tachycardia. EPS revealed a left posterolateral AP mediating an orthodromic AVRT with functional LBBB. In our patient, the late-onset manifestation of WPW syndrome with an intermittent AP were likely due to electrophysiological ageand drug-related changes in the atrioventricular node and/or the $\mathrm{AP} \cdot{ }^{[3,4]} \mathrm{CA}$ of the $\mathrm{AP}$ is the treatment of choice in patients with WPW and a fast anterogradely conducting AP and in those symptomatic for palpitations and poorly tolerated and/or drug-refractory AVRT, ${ }^{[1]}$ even when performed in elderly patients. ${ }^{[5]}$ Furthermore, in this cohort, a transseptal approach targeting the atrial insertion of the AP is preferable to a retrograde trans-aortic approach because of a high prevalence of concomitant atherosclerotic vascular disease with an associated higher risk of embolic events. ${ }^{[6]}$

\section{Conclusion}

Radiofrequency CA of left AP by transseptal approach was effective and safe for treating AVRT in an 80-year-old patient with late-onset WPW syndrome.

\section{CONFlicts OF InTEREST Disclosure}

The authors declare that they have no competing interests.

\section{ACKNOWLEDGEMENTS}

MSN certifies that SB, MC, SC and SC and he has no financial potential conflicts of interest. The idea of the case report was conceived and executed by MSN and his team. All authors participated in the management of this patient. The first author prepared the manuscript. All authors revised the paper and approved the final draft. 


\section{REFERENCES}

[1] Page RL, Joglar JAA, Caldwell MA, et al. 2015 ACC/AHA/HRS Guideline for the Management of Adult Patients With Supraventricular Tachycardia: A Report of the American College of Cardiology/American Heart Association Task Force on Clinical Practice Guidelines and the Heart Rhythm Society. J Am Coll Cardiol. 2015.

[2] Coumel P, Attuel P. Reciprocating tachycardia in overt and latent preexcitation: influence of functional bundle branch block on the rate of the tachycardia. Eur J Cardiol. 1974; 1: 423-427. PMid:4415492

[3] Brembilla-Perrot B, Yangni N'Da O, Huttin O, et al. Wolff-ParkinsonWhite syndrome in the elderly: clinical and electrophysiological findings. Arch Cardiovasc Dis. 2008; 101: 18-22. http://dx. doi .org /10.1016/S1875-2136(08) 70250-X
[4] Fan W, Peter CT, Gang ES, et al. Age-related changes in the clinical and electrophysiologic characteristic of patient with Wolff-ParkinsonWhite: comparative study between young and elderly patients. Am Heart J. 1991; 122: 741-747. http://dx.doi .org/10.1016/000 2-8703(91) 90520-R

[5] Kreiner G, Heinz G, Siostrzonek P, et al. Management of elderly patients with the Wolff-Parkinson-White syndrome: is less aggressive treatment justified? Clin Investig. 1993; 71: 519-23. PMid:8374243

[6] Dreifus LS, Pollak SJ. Ablation therapy of supraventricular tachycardia in elderly persons. Am J Geriatr Cardiol. 2005; 14 (1): 20-5. http://dx.doi.org/10.1111/j.1076-7460.2005.03339.x 